\title{
Histopathology of Intestinal Transplant Rejections
}

\author{
Tatsuaki Tsuruyama ${ }^{1,2 *}$ \\ ${ }^{1}$ Department of Pathology, Graduate School of Medicine, Kyoto University, Yoshida-konoe-cho, 606-8501 Kyoto, Japan \\ ${ }^{2}$ Center for Anatomical, Pathological, and Forensic Medical Research, Graduate School of Medicine, Kyoto University, Yoshida-konoe-cho, Kyoto 606-8501, Japan
}

Received: June 16, 2016; Accepted: July 06, 2016; Published: July 13, 2016

*Corresponding author: Tatsuaki Tsuruyama, Department of Pathology, Graduate School of Medicine, Kyoto University, Yoshida-konoe-cho, 606-8501 Kyoto, Japan

\begin{abstract}
Absract
Small bowel transplantation is one of the standard therapies for short-bowel syndrome. Nevertheless, histological rejection is still a main cause for failure of intestinal transplants. The present review aims to elucidate the histologic findings for diagnosis of acute cellular rejection (ACR). We review immunohistologic findings along with assessment of patients' clinical courses. In addition to crypt apoptosis, which is considered as a sensitive histologic finding in ACR, T-lymphocyte apoptosis and phagocytosis of apoptotic bodies in the lamina propria of villi were common findings of ACR. Recent research in variable $\mathrm{T}$ cell populations may contribute to the immunological understanding of ACR. Therefore, in the future, earlier diagnosis of ACR may be achievable.
\end{abstract}

\section{Introduction}

Small Bowel Transplantation (SBT) is one of the standard therapies for patients with complications of irreversible requirement of total parenteral nutrition associated with ShortBowel Syndrome, which is a serious condition with considerable morbidity, because of the deficiencies and metabolic imbalances created when deprived from a regular diet [1-3]. Moreover, SBT is a suitable treatment option for patients with Hirschsprung's disease and related diseases such as chronic idiopathic intestinal pseudo-obstruction syndrome [1], megacystis microcolon intestinal hypoperistalsis syndrome [4], desmoid tumors associated with familial adenomatous polyposis [5], and Crohn's disease [6]. Owing to highly effective immunosuppressive medication and improvements in post-operative care, outcomes of SBT have considerably improved $[7,8]$. For prolonged posttransplantation control, novel immunologic suppressants, such as mycophenolate mofetil, tacrolimus, and steroids have been used.

Despite improvements in the outcomes, acute cellular rejection (ACR) remains the major cause of intestinal graft failures following SBT [9-12]. For most patients that experience severe ACR, adequate recovery of mucosal function is difficult; bacterial and viral opportunistic infections are inevitable because of defensive mucosal barriers. Therefore, diagnosing ACR in the early phase is essential during postoperative care [13].

\section{Microorganisms and ACR}

The intestine is host to bacterial and microorganism flora, and its mucosa includes its native lymphoid tissue. These complicated factors contribute to the high frequency and severity of ACR. Recent studies have indicated novel techniques for identification of the flora, using profiling as a diagnostic marker of rejection [14]. Cytomegalovirus (CMV) and Epstein Barr virus (EBV) [15] infection are also causes of implications following an increased dose of steroid pulse, tacrolimus, and other immunosuppressive reagents. In particular, CMV enteritis is persistent and erosion in an immunocompromised status leads to the destruction of the mucosal architecture after severe inflammation. Immunohistochemical assessment is one of the available methods for identification of CMV. Recently, a DNA extraction technique has been developed and PCR tests for the identification of CMV have become available using formalinfixed, paraffin-embedded (FFPE) tissue [16]. The mucosal damage in CMV intestinal enteritis is frequently severe and it is difficult to make a differential diagnosis from ACR. In an immunocompromised state, patients are at increased risk of post-transplantation lymphoproliferative disorders (PTLDs) due to EBV infection. Monomorphic PTLDs have potential to develop into $\mathrm{B}$ cell lymphomas, and reduction of immunosuppression results in normalization and loss of EBV-associated expression. The expression of EBV can be analyzed by real-time PCR, and immunohistochemistry of LMP-1 or in situ hybridization for EBER (EBV encoded small RNA) [17]. Ramos, et al. [18] reported that the frequency of graft removal due to EBV infection is higher than $40 \%$, and the subsequent patient survival rate is less than $70 \%$.

\section{Candidate biomarkers of ACR}

The candidate biomarkers of ACR have been investigated in peripheral blood of intestinal transplant patients, in which ribosomal proteins such as RPL13A [3], markers IL1R2, ICAM1, GZMB, CCL3 [19,20], and citrulline levels [21] have been reported.

The production of IL-5 increases significantly relative to other cytokines in the allograft tissue during ACR [22]. In parallel with this, eosinophil infiltrates have frequently been observed [22], as well as mixed cellular inflammation [11]. C - reactive protein (CRP) is another indicator of ACR (Figure $1 \mathrm{~A}$ ); this protein is known to rise in inflammation following IL-6 secretion by macrophages. A CRP test has been shown to measure $1.0-3.0 \mathrm{mg} / 10^{-1} \mathrm{~L}$ in patients without administration of an immunosuppressive reagent; 
however, this value elevates to over $3.0 \mathrm{mg} / 10^{-1} \mathrm{~L}$ at the onset of ACR. Following immunosuppressive therapy, this value promptly decreased to $1.0 \mathrm{mg} / 10^{-1} \mathrm{~L}[22,23]$. Nonetheless, low copy numbers of CMV infection in the transplants did not significantly elevate the CRP value. Therefore, CRP value is a differential diagnostic maker of ACR from CMV enteritis.

\section{Histologic assessment of apoptotic bodies in crypts and lamina propria of villi}

Various laboratory tests have been developed for assessment of ACR; however, the significance of histologic tests remains important. Histologic criteria are shown in Table. According to these criteria, lymphocytic infiltrates (Figure 2A) and crypt apoptosis are commonly observed in ACR (Figure 2B). In practice, the diagnosis of intestinal ACR in the early phase is frequently difficult, particularly due to the complicated interactions between lymphocytes and other immunological cells resulting from the transient coexistence of donor-derived and recipient-derived cells in the graft.

Among histologic criteria, crypt apoptosis in the mucosa is one of the most reliable observations; severe ulceration follows this and sufficient recovery of the mucosa becomes difficult when graft damage reaches the submucosal area [13]. Repeat occurrences of this severe damage leads to chronic graft rejection in which fibrosis proceeds leading to irreversible inefficiency of absorption in the intestine. Apoptosis in the crypt is detectable using the TUNEL method or by the caspasecleaved keratin fragment marker.

In addition, Tsuruyama, et al. [10] reported that apoptotic bodies cluster in the Lamina Propria (LP) of villi at the onset of ACR (Figure 2C). Apoptotic crypts in grafts undergoing ACR are shown as intensely stained. In addition, macrophages phagocytosing apoptotic T cells are frequently observed with clustering in the LP (Figure 2D). These apoptotic cell clusters are significantly decreased following steroid pulse administration [10]. Therefore, this apoptotic response in LP is one of the immunological reactions associated with ACR. Scoring of the degree of apoptosis in the LP is available for evaluation (score 0 , no signals; score 1 , scant and isolated signals; score 2 , a few signal aggregations; score 3, signal aggregates surrounding

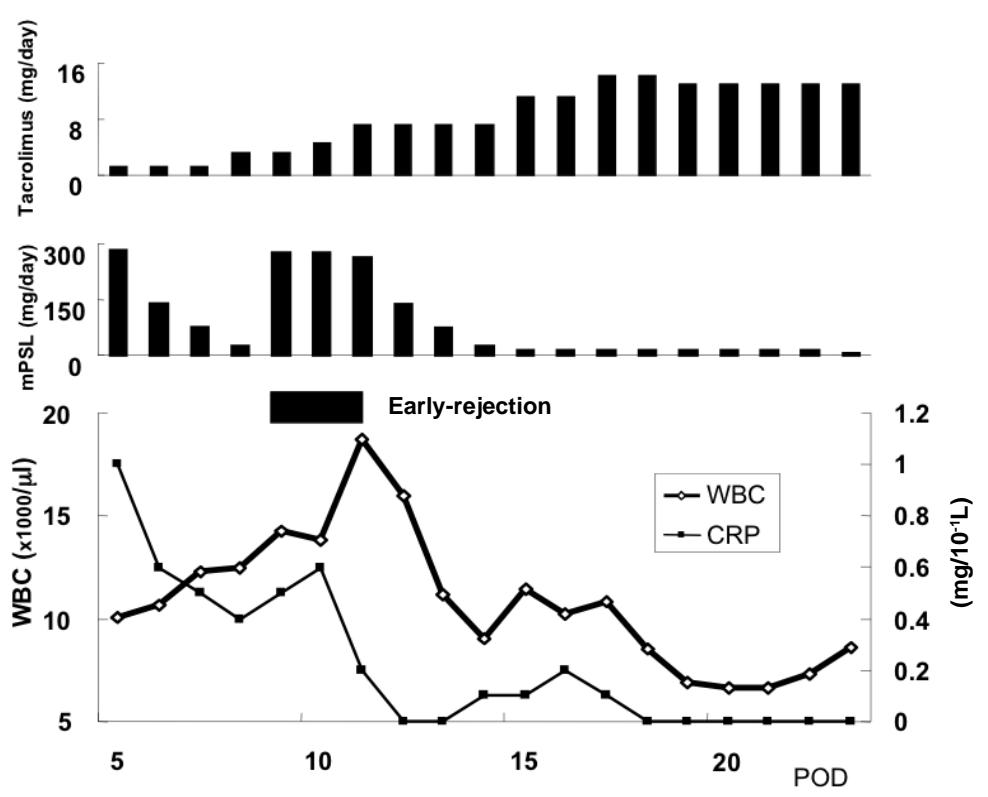

Figure 1: Clinical course of post-transplantation status. Administrative doses of tacrolimus and methylpredinisolone (mPSL). Bottom plots represent white blood cell (WBC) number and concentration of C-reactive protein (CRP) in the peripheral blood.

Table 1: Histologic schema of acute cellular rejection (ACR) of intestinal allograft.

\begin{tabular}{|l|l|l|}
\hline Histologic grade & Crypt apoptosis and related findings & Lymphocytic apoptosis in LP \\
\hline Indeterminate & up to 6 apoptotic bodies per 10 crypts & None \\
\hline Mild & $>6$ apoptotic bodies per 10 crypts & Isolated apoptotic bodies \\
\hline Moderate & $\begin{array}{l}\text { Confluent apoptosis } \\
\text { Increased Inflammation, Epithelial injury }\end{array}$ & A few apoptotic body cluster \\
\hline Severe/ Exfoliative & Mucosal ulceration & Apoptotic bodies aggregate \\
\hline LP: Lamina Propria & & \\
\hline
\end{tabular}




\section{A}

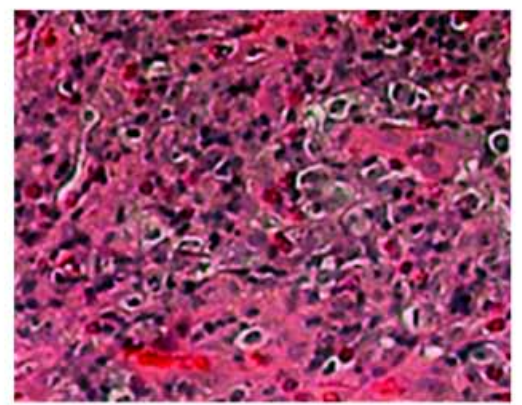

B

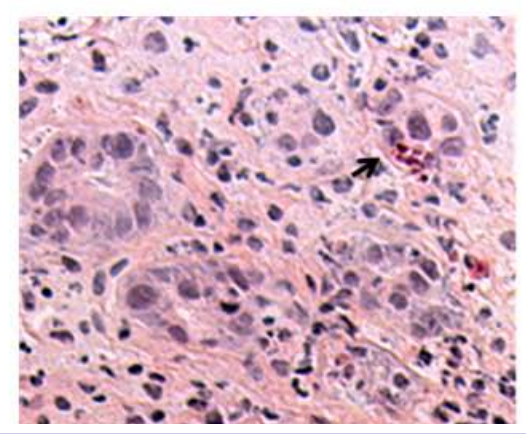

$\mathrm{C}$

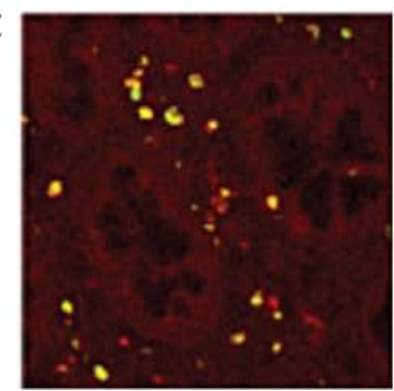

D

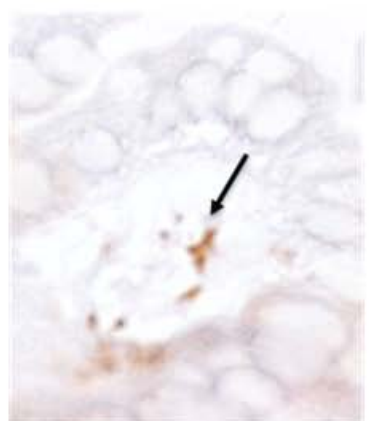

Figure 2: Histology of ACR in the intestine.

A) Lymphocyte infiltrates including eosinophils and neutrophils $(\times 200)$.

B) Crypt apoptosis $(\times 200)$. An Arrow indicates the eosinophilic apoptotic bodies in the crypt.

C) Double staining of NKT cells with TCRValpha24 (red, PE) and TUNEL (green, FITC) $(\times 200)$.

D) Apoptotic clusters stained with TUNEL. DAB was used for visualization. An arrow represents the cluster.

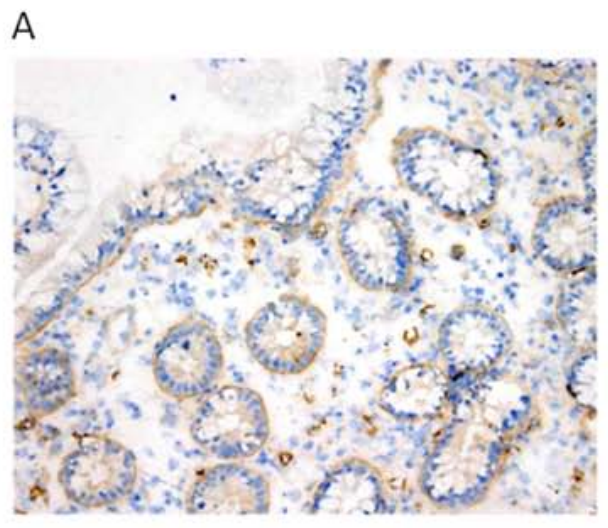

B

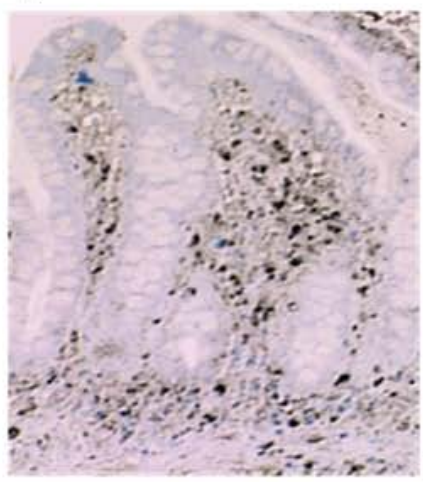

Figure 3: Immunohistochemistry of FasL. A) Intact allograft $(\times 200)$, and B) allograft with ACR $(\times 200)$. DAB was used for visualization. FasLpositive cells were observed in the lamina propria.

the crypt) [11]. The T cell apoptosis score closely correlates with the crypt apoptosis count. In addition, the receptor Fas is also available for Immunostaining for the identification of apoptosis [10]. It is probable that the FasL-Fas interaction contributes to the apoptosis reaction in LP. Fas Ligand (FasL) staining is useful for the identification of apoptotic bodies in allograft (Figure 3A, B).

Asaoka, et al. [20] reported the activation of cytotoxic T cells (CTLs) in granzyme B/ perforin-mediated graft injury. Unlike apoptosis in graft versus host disease that may be associated with elevation in TNF-alpha production [24], Fas ligand (FasL) is frequently stained in cases of ACR [10]. Therefore, the ACR of intestinal graft includes various activations of apoptosis-related molecules, by expression and release. After $\mathrm{T}$ cells undergo apoptosis [10], apoptotic bodies are then phagocytosed by macrophages. Similar findings in a liver allograft have been reported [25]. Thus, these phagocytic findings may be common to multiple allografts in ACR.

\section{Time course of apoptosis in ACR and recovery of the mucosa}

To our experience, crypt apoptosis and mixed cellular infiltrates appear at the late stage of ACR of small intestine and are irreversible changes. Once crypt apoptosis develops, even 
after steroid pulse, apoptosis increases in number and ulceration is inevitable. In contrast, T cell apoptosis in LP appears in the early phase of ACR and immunosuppressive therapy is sufficiently effective, and in fact, apoptotic $\mathrm{T}$ cells disappear within a day following the therapy $[10,22]$. For this reason, $\mathrm{T}$ cell apoptosis is more sensitive to treatment procedures. Thus, CRP test and histology likely provides effective prospects for follow-up. After treatment of rejection ACR erosive mucosa is edematous; however, Paneth cells at the crypt regenerate in association with mucosal recovery. Cell cycle markers, such as Ki67 and PCNA, show recovery of stainability in foveolar and crypt epithelial cells. Therefore, this staining method provides a marker of better prognosis after ACR. Nonetheless, for diagnosis of cases in which humoral rejection is suspected, complement staining such as $\mathrm{C} 4 \mathrm{~d}$ using frozen section may provide more reliable information [12].

\section{Histologic finding in Peyer's patch (PP) and Isolated Lymphoid Follicle (ILF)}

After engraftment, host-derived T cells traffic into the intestinal allograft across the high endothelial veins (HEVs) located in the inter-PP follicular region. By endoscopy examination, elevation of mucosa is frequently observed in the intestinal transplant at the onset of ACR. The elevated site includes PPs that consist of B cells, T cells, and dendritic Cells.

The entry of cytotoxic T cells CTLs into PPs via HEVs is observed within 7-10 days after transplantation and ACR occurs in cases. When ACR persists, PPs disintegrate in severe ACR and mucosal recovery is not sufficient when fibrosis develops. This is one of the poor prognoses of intestinal transplants, because once the mucosal defensive mechanism is lost in the erosive site, the graft becomes susceptible to bacterial and viral infection. Therefore, the immunosuppressive therapy before PP disintegration is essential for the control of post-transplantation success rate [23].

Follicular B cells in PPs are stimulated by antigens in the lumen and differentiate from Immunoglobulin $\mathrm{M}(\operatorname{IgM}+)$ to IgA+ B cells by class switching, which is mediated by Activation-Induced Cytidine Deaminase (AID). IgA+ B cells in PPs circulate throughout the body via the thoracic duct and differentiate into IgA+ B cells by the effect of IL-6 produced by intestinal epithelial cells. The primary antibodies secreted into the intestinal tract mucus are of the IgA class, and are transported to the gut luminal side by binding to multimeric antibody receptors that are retained on intestinal epithelial cells. Notably, host lymphocytes rapidly repopulate allograft PPs/ILFs within two years in the absence of ACR [26]. Allograft ILFs revealed a higher maturation state than control samples, and IgA+ plasma cells were increased in a number in allograft mucosa [26]. AID gene expression in allograft PPs/ILFs that the immunological burden may promote the maturation of B cells [26]. Histological examination showed hyperplastic changes of PPs with an increase in expression of CD20, a mature B cell marker at the onset of ACR [23].

\section{T cells infiltrate allografts at the onset of ACR}

It appears to be controversial which types of lymphocytes induce ACR of intestinal allografts. In general, CTLs are considered to induce ACR of intestinal allografts. However, several experimental studies have not supported this. In fact, apoptosis and ACR of intestinal allograft was observed in the absence of CTLs in a rat model [27]. In fact, our immunohistochemistry did not consistently reveal CTL infiltrates in allografts except in the first episode of rejection $[10,22,23,28]$. CTL has the potential to express FasL. In autoimmune diseases such as inflammatory bowel disease (IBD) such as ulcerative colitis (UC), increases in FasL+ T cells are observed, but do not correlate with increases in Fas+ T cells, indicating that the increased expression of FasL in IBD colonic LP is not paralleled by Fas expression by $\mathrm{T}$ cells, and that Fas / FasL-mediated apoptosis is not the main factor. In contrast, expression of perforin, which is another apoptosis-inducing molecule, is correlated with tissue damage and may represent the enhancement of a distinct cytotoxic pathway in UC [29]. Unlike UC, Fas and FasL expression correlates well with ACR in intestinal grafts. Therefore, the immunological status is probably not similar to autoimmune colitis. Then, which types of $\mathrm{T}$ cells expressing FasL, or other cell populations, are effectors in ACR of intestinal allografts? The helper T cell (Th)1/ Th2 paradigm in mucosal immunology has been shift-
A

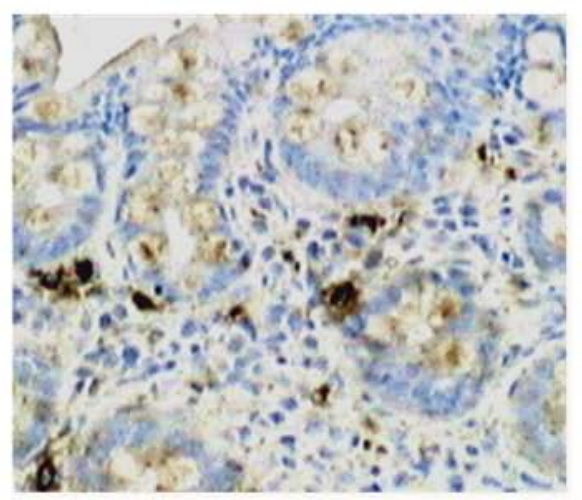

B

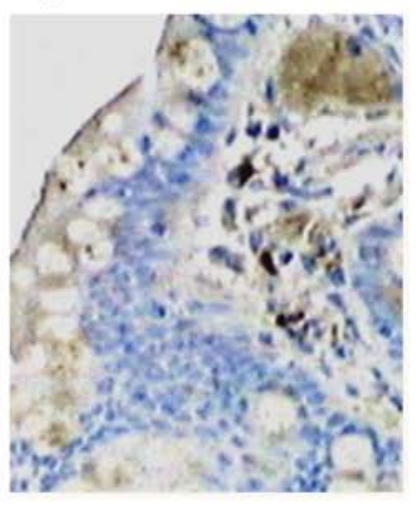

Figure 4: Immunohistochemistry of the restricted T cell receptor (TCR) repertoire. TCR beta11 subunit was stained for detection of NKT cells in the lamina propria of villi $(A, \times 200$; and $B, \times 200)$. NKT cells were observed in the lamina propria. 
ed [30], and recent discovery of novel subsets of natural killer $\mathrm{T}$ (NKT) cells, regulatory $\mathrm{T}$ (Treg) cells $[31,32]$ and effecter $\mathrm{T}$ helper cells that produce interleukin (IL)-17 (Th17) have been reported [31]. Of note, Th17 cells are potent inducers of inflammation and autoimmune diseases.

At the onset of ACR, natural killer (NK) cells and natural killer $\mathrm{T}$ (NKT) cells transiently increase in number, as well as iNKT cells (Figure 4A, B), and both rapidly decrease following steroid pulse therapy [23]. The iNKT cells have the potential to produce IL-4, which contributes to the development of Th2 cells and antagonizes Th1 and CTL responses. Higher levels of IL-4 prior to and shortly after kidney transplantation have been reported, and IL-4 may have a protective effect on renal graft survival [33]. Indeed, NKT cells have been implicated in allograft tolerance in experimental mouse models [34], in induction of chimerism in allogeneic cardiac transplant models [35], and in acceptance of rat-islet xenografts in mice [36]. Interferon- $\gamma$ production remains low relative to normal donor intestine and does not change during the course of ACR development [37]. Therefore, infiltration of NKT cells may be involved in the protection of allografts in the response to ACR. Therefore, release of Th2-related cytokines by NKT cells may antagonize the proceeding of ACR. It is likely that humoral factors, such as candidates IL-10 and transforming growth factor (TGF)- $\beta$ [23], recruit NKT cells to the graft mucosa in order to suppress allograft ACR. Using TUNEL and restricted-T cell receptor (TCR) alpha 24 staining, NKT cells are found to increase and undergo apoptosis (Figure 2C). Therefore, the apoptotic reaction involves the NKT cell population. Decrease of NKT cells may lead to the deterioration of ACR. However, because the released IL-4 and IL-5 may damage the allograft via eosinophilic enteritis, NKT cells are a double-edged effector [38].

FoxP3+ Treg are another immunological modulator of intestinal allograft, and the graft-protective mechanism has been extensively investigated [39]. Treg are recruited to the liver allograft at the onset of ACR and are maintained in tolerated liver allograft [40]. Introduction of bone marrow mesenchymal cells into the intestinal allograft increased Treg in parallel with IL-10 and TGF- $\beta$ [41]. In clinical cases, the roles of Treg are not understood with respect to tolerance or induction of ACR of intestinal allografts. In experimental studies linked with human biopsy samples, IL-17 plays a critical role in ACR of intestinal transplantation [37,42], and may be a target for inhibiting ACR.

\section{Conclusion}

ACR remains to be the main cause of intestinal graft failure. Research in molecular immunological responses by $\mathrm{T}$ cell populations in allograft has developed, and a greater understanding of the pathogenesis of ACR is expected in the future. With respect to these achievements, histologic diagnosis of rejection at an earlier phase should be possible.

\section{References}

1. Bond GJ, Reyes JD. Intestinal transplantation for total/neartotal aganglionosis and intestinal pseudo-obstruction. Semin Pediatr Surg. Semin Pediatr Surg. 2004;13(4):286-92.
2. Reyes J. Intestinal transplantation for children with short bowel syndrome. Semin Pediatr Surg. 2001;10(2):99-104.

3. Pirenne J, Hoffman I, Miserez M, Coosemans W, Aerts R, Monbaliu D, et al. Selection criteria and outcome of patients referred to intestinal transplantation: an European center experience. Transplant Proc. 2006;38(6):1671-2.

4. Masetti M, Rodriguez MM, Thompson JF, Pinna AD, Kato $\mathrm{T}$, Romaguera RL, et al. Multivisceral transplantation for megacystis microcolon intestinal hypoperistalsis syndrome. Transplantation. 1999;68(2):228-32.

5. Chatzipetrou MA, Tzakis AG, Pinna AD, Kato T, Misiakos $\mathrm{EP}, \mathrm{Tsarouch} \mathrm{AK}$, et al. Intestinal transplantation for the treatment of desmoid tumors associated with familial adenomatous polyposis. Surgery. 2001;129(3):277-81.

6. Gerlach UA, Vrakas G, Reddy S, Baumgart DC, Neuhaus P, Friend PJ, et al. Chronic intestinal failure after Crohn disease: when to perform transplantation. JAMA Surg. 2014;149(10):1060-6.

7. Misiakos EP, Weppler D, Bakonyi A, Nery JR, Pinna AD, Kato $\mathrm{T}$, et al. Clinical outcome of intestinal transplantation at the University of Miami. Transplant Proc. 1999;31(1-2):569-71.

8. Kato T, Nishida S, Mittal N, Levi D, Nery J, Madariaga J, et al. Intestinal transplantation at the University of Miami. Transplant Proc. 2002;34(3):868.

9. Ruiz P, Bagni A, Brown R, Cortina G, Harpaz N, Magid MS, et al. Histological criteria for the identification of acute cellular rejection in human small bowel allografts: results of the pathology workshop at the VIII International Small Bowel Transplant Symposium. Transplant Proc. 2004;36(2):335-7.

10. Tsuruyama T, Okamoto S, Fujimoto $\mathrm{Y}$, Yoshizawa A, Yoshitoshi E, Egawa $\mathrm{H}$, et al. Histology of intestinal allografts: lymphocyte apoptosis and phagocytosis of lymphocytic apoptotic bodies are diagnostic findings of acute rejection in addition to crypt apoptosis. Am J Surg Pathol. 2013;37(2):178-84.

11. Wu T, Abu-Elmagd K, Bond G, Nalesnik MA, Randhawa P, Demetris AJ, et al. A schema for histologic grading of small intestine allograft acute rejection. Transplantation. 2003;75(8):1241-8.

12. Yoshitoshi EY, Yoshizawa A, Ogawa E, Kaneshiro M, Takada $\mathrm{N}$, Okamoto $\mathrm{S}$, et al. The challenge of acute rejection in intestinal transplantation. Pediatr Surg Int. 2012;28(8):8559. doi: 10.1007/s00383-012-3110-x.

13. Selvaggi G, Gaynor JJ, Moon J, Kato T, Thompson J, Nishida S, et al. Analysis of acute cellular rejection episodes in recipients of primary intestinal transplantation: a single center, 11year experience. Am J Transplant. 2007;7(5):1249-57.

14. Oh PL, Martinez I, Sun Y, Walter J, Peterson DA, Mercer DF, et al. Characterization of the ileal microbiota in rejecting and nonrejecting recipients of small bowel transplants. Am 
J Transplant. 2012 Mar;12(3):753-62. doi: 10.1111/j.16006143.2011.03860.x.

15. Lauro A, Pinna AD, Pellegrini S, Bagni A, Zanfi C, Dazzi A, et al. Long-term endoscopic follow-up in small bowel transplant recipients: single-center series. Transplant Proc. 2014;46(7):2325-8.

16. Mills AM, Guo FP, Copland AP, Pai RK, Pinsky BA, et al. A comparison of CMV detection in gastrointestinal mucosal biopsies using immunohistochemistry and PCR performed on formalin-fixed, paraffin-embedded tissue. Am J Surg Pathol. 2013;37(7):995-1000.

17. Sarkar S, Selvaggi G, Mittal N, Cenk Acar B, Weppler D, Kato $\mathrm{T}$, et al. Gastrointestinal tract ulcers in pediatric intestinal transplantation patients: etiology and management. Pediatr Transplant. 2006;10(2):162-7.

18. Ramos E, Hernandez F, Andres A, Martinez-Ojinaga E, Molina M, SarriaJ, etal. Post-transplantlymphoproliferative disorders and other malignancies after pediatric intestinal transplantation: incidence, clinical features and outcome. Pediatr Transplant. 2013;17(5):472-8. doi: 10.1111/petr.12103.

19. Andreev VP, Tryphonopoulos P, Blomberg BB, Tsinoremas N, Weppler D, Neuman DR, et al. Peripheral blood gene expression analysis in intestinal transplantation: a feasibility study for detecting novel candidate biomarkers of graft rejection. Transplantation. 2011;92(12):1385-91. doi: 10.1097/ TP.0b013e3182370db1.

20. Asaoka T, Island ER, Tryphonopoulos P, Selvaggi G, Moon J, Tekin A, et al. Characteristic immune, apoptosis and inflammatory gene profiles associated with intestinal acute cellular rejection in formalin-fixed paraffin-embedded mucosal biopsies. Transpl Int. 2011;24(7):697-707. doi: 10.1111/j.1432-2277.2011.01259.x.

21. David AI, Selvaggi G, Ruiz P, Gaynor JJ, Tryphonopoulos P, Kleiner GI, et al. Blood citrulline level is an exclusionary marker for significant acute rejection after intestinal transplantation. Transplantation. 2007;84(9):1077-81.

22. Tsuruyama T, Fujimoto Y, Yonekawa Y, Miyao M, Onodera $\mathrm{H}$, Uemoto $\mathrm{S}$, et al. Invariant natural killer $\mathrm{T}$ cells infiltrate intestinal allografts undergoing acute cellular rejection. Transpl Int. 2012;25(5):537-44.

23. Tsuruyama T, Aini W. The roles of invariant NKT cells in bowel immunity: suppression of tumor progression and rejection of intestinal transplant. "Immune Response Activation". edited by Guy Huynh Thien Duc, chapter 8, 2014: p. 227-45.

24. Stuber E, Buschenfeld A, von Freier A, Arendt T, Folsch UR. Intestinal crypt cell apoptosis in murine acute graft versus host disease is mediated by tumour necrosis factor alpha and not by the FasL-Fas interaction: effect of pentoxifylline on the development of mucosal atrophy. Gut. 1999;45(2):229-35.

25. Miyagawa-Hayashino A, Tsuruyama T, Egawa H, Haga $H$, Sakashita H, Okuno T, et al. FasL expression in hepatic antigen- presenting cells and phagocytosis of apoptotic $\mathrm{T}$ cells by FasL+ Kupffer cells are indicators of rejection activity in human liver allografts. Am J Pathol. 2007;171(5):1499-508.

26. Meier D, Docena GH, Ramisch D, Toscanini U, Berardi G, Gondolesi GE, et al. Immunological status of isolated lymphoid follicles after intestinal transplantation. Am J Transplant. 2014;14(9):2148-58.

27. Ogura Y, Martinez OM, Villanueva JC, Tait JF, Strauss HW, Higgins JP, et al. Apoptosis and allograft rejection in the absence of CD8+ T cells. Transplantation. 2001;71(12):182734 .

28. Fujimoto Y, Uemoto S, Inomata Y, Egawa H, Fujita S, Kawanami T, et al. Small bowel transplantation using grafts from living-related donors. Two case reports. Transpl Int. 2000;13 Suppl 1:S179-84.

29. Souza HS, Tortori CJ, Castelo-Branco MT, Carvalho AT, Margallo VS, Delgado CF, et al. Apoptosis in the intestinal mucosa of patients with inflammatory bowel disease: evidence of altered expression of FasL and perforin cytotoxic pathways. Int J Colorectal Dis. 2005;20(3):277-86.

30. Neurath MF, Finotto S, Glimcher LH. The role of Th1/Th2 polarization in mucosal immunity. Nat Med. 2002;8(6):56773.

31. Bettelli E, Carrier Y, Gao W, Korn T, Strom TB, Oukka M, et al. Reciprocal developmental pathways for the generation of pathogenic effector TH17 and regulatory T cells. Nature. 2006;441(7090):235-8.

32. La Cava A, Van Kaer L, Fu Dong S. CD4+CD25+ Tregs and NKT cells: regulators regulating regulators. Trends Immunol. 2006;27(7):322-7.

33. Karczewski J, Karczewski M, Glyda M, Wiktorowicz K. Role of TH1/TH2 cytokines in kidney allograft rejection. Transplant Proc. 2008;40(10):3390-2.

34. Jiang X, Kojo S, Harada M, Ohkohchi N, Taniguchi M, Seino KI. Mechanism of NKT cell-mediated transplant tolerance. Am J Transplant. 2007;7(6):1482-90.

35. Seino KI, Fukao K, Muramoto K, Yanagisawa K, Takada Y, Kakuta S, et al. Requirement for natural killer T (NKT) cells in the induction of allograft tolerance. Proc Natl Acad Sci U S A. 2001;98(5):2577-81.

36. Liu Y, Luan X, Li J, He Y, Li M. The role of invariant NKT cells in liver transplant tolerance in rats. Transplant Proc. 2012;44(4):1041-4.

37. Yang JJ, Feng F, Hong L, Sun L, Li MB, Zhuang R, et al. Interleukin-17 plays a critical role in the acute rejection of intestinal transplantation. World J Gastroenterol. 2013;19(5):682-91.

38. Lisbonne M, Leite-de-Moraes MC. Invariant Valpha14 NKT lymphocytes: a double-edged immuno-regulatory $\mathrm{T}$ cell population. Eur Cytokine Netw. 2003;14(1):4-14. 
39. Ceulemans LJ, Braza F, Monbaliu D, Jochmans I, De Hertogh G, $\mathrm{Du}$ Plessis J, et al. The Leuven immunomodulatory protocol promotes T-regulatory cells and substantially prolongs survival after first intestinal transplantation. Am J Transplant. 2016.

40. Li Y, Zhao X, Cheng D, Haga H, Tsuruyama T, Wood K, et al. The presence of Foxp3 expressing $\mathrm{T}$ cells within grafts of tolerant human liver transplant recipients. Transplantation. 2008;86(12):1837-43.
41. Yang Y, Song HL, Zhang W, Wu BJ, Fu NN, Zheng WP, et al. Reduction of acute rejection by bone marrow mesenchymal stem cells during rat small bowel transplantation. PLoS One. 2014;9(12):e114528.

42. Acosta-Rodriguez EV, Napolitani G, Lanzavecchia A, Sallusto F. Interleukins 1 beta and 6 but not transforming growth factor-beta are essential for the differentiation of interleukin 17-producing human $\mathrm{T}$ helper cells. Nat Immunol. 2007;8(9):942-9. 